$\mathcal{F}\left\langle\eta_{1}, \cdots, \eta_{n}\right\rangle$ is contained in a liouvillian extension of $\mathcal{F}$ of the required type. It follows that $\eta_{1}, \cdots, \eta_{n}$ is a fundamental system of solutions of $L(y)$, and (see PV, §25) $\mathcal{F}\left\langle\eta_{1}, \cdots, \eta_{n}\right\rangle$ is itself a liouvillian extension of $\mathcal{F}$ of the required type.

Columbia University

\title{
ON SOME EXAMPLES IN THE THEORY OF POWER SERIES
}

\section{P. TURÁN}

Fabry, ${ }^{1}$ Hardy, ${ }^{2}$ S. Bernstein ${ }^{3}$ and Carleman ${ }^{4}$ discovered that for any $\delta>0$ there exist power series $\sum_{0}^{\infty} a_{v} z^{\nu}$ which are continuous for $|z| \leqq 1$ and for which the series $\sum\left|a_{\nu}\right|^{2-\delta}$ diverges. An elegant example is provided by the series

$$
f(z)=\sum_{n=2}^{\infty} \frac{e^{i c n \log n}}{n^{1 / 2} \log ^{\beta} n} z^{n}, \quad c \neq 0, \beta>1,
$$

which is continuous for $|z| \leqq 1$ (and even uniformly convergent there) $;^{5}$ another example for the Carleman singularity, explicitly

Received by the editors July 22, 1947, and, in revised form, November 17, 1947.

${ }^{1}$ E. Fabry, Ordre des points singuliers de la série de Taylor, Acta Math. vol. 36 (1913) pp. 69-194, esp. p. 103.

${ }^{2}$ G. H. Hardy, A theorem concerning Tayor's series, Quarterly Journal of Pure and Applied Mathematics vol. 44 (1913) pp. 147-160. In these two papers it was shown that if $\delta>0$ the series $f_{2}(z)=\sum_{1}^{\infty} e^{i \nu^{1-\delta} / z^{\nu} / \nu^{1-\delta}}$ is uniformly convergent for $|z|<1$ and in Hardy's paper remark was made (p. 157) upon H. Bohr's problem on constructing a power series which is uniformly but not absolutely convergent for $|z| \leqq 1$.

${ }^{3}$ S. Bernstein, C. R. Acad. Sci. Paris (1914). He gave interesting cosine-polynomials $H(x)=\sum_{\nu=1}^{p-1} b_{\nu} \cos \nu x(p$ prime $\equiv 1 \bmod 4)$ with the properties $|H(x)| \leqq 1$ and $\sum_{\nu=1}^{p=1}\left|b_{\nu}\right|=(p-1) / p^{1 / 2}$ which contains the seeds of the Carleman-singularity and were indeed the basis of Carleman's own construction.

${ }^{4} \mathrm{~T}$. Carleman, Über Fourier Koefficienten einer steigen Funktionen, Acta. Math. vol. 41 (1918) pp. 337-384. Here is asserted explicitly and proved for the first time the existence of a continuous $f_{3}(x) \sim \sum\left(a_{\nu} \cos \nu x+b_{\nu} \sin \nu x\right)$ with $\sum_{1}^{\infty}\left(\left|a_{\nu}\right|^{2-\delta}+\left|b_{\nu}\right|^{2-\delta}\right)$ $=\infty, \delta$ arbitrarily small: the existence of a continuous power series with the same property seems to be explicitly mentioned at the first time by Sidon; see footnote 9.

${ }^{5}$ See G. H. Hardy and J. E. Littlewood (Some problems of Diophantine approximation: A remarkable trigonometrical series, Proc. Nat. Acad. Sci. U.S.A. vol. 2 (1916) pp. $583-586)$, who considered only the functions $f_{1}(z)=\sum_{n-2}^{\infty}\left(e^{i c n} \log n / n^{1 / 2+\alpha}\right) z^{n}, c \neq 0$, $0<\alpha<1$ (the divergence of the $(2-\delta)$ th power of the moduli of the coefficients is not explicitly mentioned there). Series (1) seems to have been discussed for the first time by Zygmund in his book on trigonometric series. He uses there (in a simplified form) an argument due to Hille (Note on a power series considered by Hardy and Littlewood, J. London Math. Soc. vol. 4 (1929) pp. 176-182) and based on the application of Van der Corput's estimates to sums of the form $\sum e^{2 \pi i g(n)}$. 
stated only for trigonometrical series, is given by Landau ${ }^{6}$ who used number-theoretical characters and interpolation. Interesting generalizations were given by Gronwall ${ }^{7}$ and Sidon $^{8}$ in the case of trigonometrical series; the first of these asserts that given any positive $\phi(x)$ with the property $\lim _{x \rightarrow 0} \phi(x)=+\infty$ then there is a continuous $f_{4}(x) \sim \sum_{\nu=0}^{\infty}\left(a_{\nu} \cos \nu x+b_{\nu} \sin \nu x\right)$ with $\sum_{\nu=0}^{\infty}\left(a_{\nu}^{2}+b_{\nu}^{2}\right) \phi\left(a_{\nu}^{2}+b_{\nu}^{2}\right)$ $=+\infty$, and according to the second to any given $\delta>0$ and sequence of positive integers $n_{1}<n_{2}<\cdots$ we can construct a continuous $f_{4}(x) \sim \sum_{0}^{\infty}\left(a_{\nu} \cos \nu x+b_{\nu} \sin \nu x\right)$ such that $\sum\left(\left|a_{n_{k}}\right|^{2-\delta}+\left|b_{n_{k}}\right|^{2-\delta}\right)=\infty$. Finally Sidon ${ }^{9}$ proved the same theorems for power series.

In what follows I shall give an entirely elementary method of constructing all the required examples except Sidon's generalization (which method works of course in elementary constructions of corresponding examples concerning the theory of Lipschitz condition). This needs no reference either to Van der Corput's important estimations or to special number-theoretical functions and perhaps it is more suitable to lectures. It will be sufficient to show the construction on the Carleman-Sidon phenomenon. Our example will be

$$
F(z)=\sum_{n=4}^{\infty} \frac{1}{n^{1+\epsilon / 3}} \sum_{\nu=n^{2}+1}^{(n+1)^{2}} e^{-i v \theta_{n} z^{\nu}}=\sum_{\nu=17}^{\infty} C_{\nu} z^{\nu}, \quad \epsilon>0,
$$

where the numbers $\theta_{n}$ are defined by

$$
\begin{array}{r}
\theta_{n}=\left(n-2^{r}\right) \frac{2 \pi}{2^{r}} \quad \text { for } n=2^{r},\left(2^{r}+1\right), \cdots,\left(2^{r+1}-1\right) ; \\
r=2,3, \cdots ;
\end{array}
$$

we suppose

$$
0<\epsilon<1 / 10
$$

Then

$$
\sum_{\nu=17}^{\infty}\left|C_{\nu}\right|^{2-\epsilon}=\sum_{n=4}^{\infty} \frac{1}{\left(n^{1+\epsilon / 3}\right)^{2-\epsilon}}(2 n+1)=+\infty .
$$

Hence we have only to show that the series (2) converges uniformly

${ }^{6}$ E. Landau, Bemerkungen zu einer Arbeit von Herren Carleman, Math. Zeit. vol. 5 (1919) pp. 147-153. The historical data are partly taken from this thorough paper.

${ }^{7}$ T. H. Gronwall, On the Fourier coefficients of a continuous function, Bull. Amer. Math. Soc. vol. 27 (1921) pp. 320-321.

${ }^{8}$ S. Sidon, Ein Satz uber trigonometrische Polynome mit Litcken, J. Reine Angew. Math. vol. 163, pp. 251-252.

9 S. Sidon, Bemerkungen iber Fourier und Potenzreihen, Acta Univ. Szeged. vol. 7 (1934-1935) pp. 85-94. 
for $|z|=1$ or, putting $z=e^{i \theta}$, uniformly in $\theta$. For the inner sum we have

$$
\begin{aligned}
\left|\sum_{\nu=n^{2}+1}^{(n+1)^{2}} e^{i \nu\left(\theta-\theta_{n}\right)}\right| & =\left|\sum_{\nu=0}^{2 n} e^{i \nu\left(\theta-\theta_{n}\right)}\right|=\left|\frac{1-e^{(2 n+1) i\left(\theta-\theta_{n}\right)}}{1-e^{i\left(\theta-\theta_{n}\right)}}\right| \\
& <\min \left(2 n+1, \frac{1}{\left|\sin \left(\left(\theta-\theta_{n}\right) / 2\right)\right|}\right) \\
& <3 \min \left(n, \frac{1}{\left|\sin \left(\left(\theta-\theta_{n}\right) / 2\right)\right|}\right) .
\end{aligned}
$$

Let us now be given an integer $\omega \geqq 17$; we determine the integer $\kappa$ by

$$
\kappa^{2}<\omega \leqq(\kappa+1)^{2} \text {. }
$$

Then putting

$$
R_{\omega}(z)=\sum_{\nu=\omega}^{\infty} C_{\nu} z^{\nu}
$$

we have

(7)

$$
\begin{aligned}
\left|R_{\omega}\left(e^{i \theta}\right)\right| & \leqq\left|\sum_{\omega<\nu \leqq(\kappa+1)^{2}} C_{\nu} z^{\nu}\right|+\left|\sum_{\nu>(\kappa+1)^{2}} C_{\nu} z^{\nu}\right| \\
& <\frac{1}{\kappa^{1+\epsilon / 3}}(2 \kappa+1)+\left|\sum_{n=\kappa+1}^{\infty} \frac{1}{n^{1+\epsilon / 3}} \sum_{\nu=n^{2}+1}^{(n+1)^{2}} e^{\nu i\left(\theta-\theta_{n}\right)}\right| \\
& <\frac{3}{\kappa^{\epsilon / 3}}+\sum_{n=\kappa+1}^{\infty} \frac{1}{n^{1+\epsilon / 3}}\left|\sum_{\nu=n^{2}+1}^{(n+1)^{2}} e^{\nu i\left(\theta-\theta_{n}\right)}\right| \\
& <3 \kappa^{-\epsilon / 3}+3 \sum_{n=\kappa+1}^{\infty} n^{-1-\epsilon / 3} \min \left(n, \frac{1}{\left|\sin \left(\left(\theta-\theta_{n}\right) / 2\right)\right|}\right)
\end{aligned}
$$

using (5). If $L$ is defined by

$$
2^{L} \leqq \kappa<2^{L+1}
$$

then from (7) and (8)

$$
\begin{aligned}
\left|R_{\omega}\left(e^{i \theta}\right)\right| & <3 \kappa^{-\epsilon / 3}+3 \sum_{n=2^{L}}^{\infty} n^{-1-\epsilon / 3} \min \left(n, \frac{1}{\left|\sin \left(\left(\theta-\theta_{n}\right) / 2\right)\right|}\right) \\
& <5 \omega^{-\epsilon / 6}
\end{aligned}
$$

$$
+3 \sum_{\nu=L}^{\infty} \sum_{2^{\nu} \leqq n<2^{\nu+1}} n^{-1-\epsilon / 3} \min \left(n, \frac{1}{\left|\sin \left(\left(\theta-\theta_{n}\right) / 2\right)\right|}\right)
$$




$$
\begin{aligned}
<5 \omega^{-\epsilon / 6} & \\
& +3 \sum_{\nu=L}^{\infty} 2^{-\nu(1+\epsilon / 3)} \sum_{2^{\nu} \leqq n<2^{\nu+1}} \min \left(2^{\nu+1}, \frac{1}{\left|\sin \left(\left(\theta-\theta_{n}\right) / 2\right)\right|}\right) \\
= & 5 \omega^{-\epsilon / 6}+3 \sum_{\nu=L}^{\infty} 2^{-\nu(1+\epsilon / 3)} S_{\nu}(\theta) .
\end{aligned}
$$

Now we consider $S_{\nu}(\theta)$. Taking in account the explicit form of the $\theta_{n}$ 's we may observe that $S_{\nu}(\theta)$ is periodical with the period $2 \pi / 2^{\nu}$, hence we may suppose $0 \leqq \theta \leqq 2 \pi / 2^{\nu}$. Then using the inequality $\sin x \geqq 2 / \pi x$ valid for $0 \leqq x \leqq \pi / 2$, we obtain

$$
\begin{aligned}
S_{\nu}(\theta) & \leqq 3 \cdot 2^{\nu+1}+\sum_{n=2^{\nu}+2}^{2 \nu+1-1} \min \left(2^{\nu+1}, \frac{1}{\left|\sin \left(\left(\theta-\theta_{n}\right) / 2\right)\right|}\right) \\
& =3 \cdot 2^{\nu+1}+\sum_{m=2}^{2^{\nu}-1} \min \left(2^{\nu+1}, \frac{1}{\left|\sin 2^{-1}\left(\theta-2 \pi m / 2^{\nu}\right)\right|}\right) \\
& \leqq 3 \cdot 2^{\nu+1}+2 \sum_{2 \leqq m \leqq 2^{\nu-1}} \min \left(2^{\nu+1}, \frac{1}{\sin 2^{-1}\left(2 \pi m / 2^{\nu}-\theta\right)}\right) \\
& \leqq 3 \cdot 2^{\nu+1}+2 \sum_{2 \leqq m \leqq 2^{\nu-1}} \min \left(2^{\nu+1}, \frac{1}{\sin \left((m-1) 2 \pi / 2^{\nu+1}\right)}\right) \\
& =3 \cdot 2^{\nu+1}+2 \sum_{1 \leqq m \leqq 2^{\nu-1}} \frac{1}{\sin \left(m 2 \pi / 2^{\nu+1}\right)} \\
& <3 \cdot 2^{\nu+1}+\frac{1}{2} \sum_{1 \leqq m \leqq 2^{\nu-1}} 2^{\nu+1} \frac{1}{m}<2^{\nu+1}(3+\nu)<4 \nu 2^{\nu} .
\end{aligned}
$$

Substituting this into (9) we obtain, using (4),

$$
\begin{aligned}
\left|R_{\omega}\left(e^{i \theta}\right)\right| & <5 \omega^{-\epsilon / 6}+3 \sum_{\nu \geqq L} 2^{-\nu(1+\epsilon / 3)} 4 \cdot \nu \cdot 2^{\nu}=5 \omega^{-\epsilon / 6}+12 \sum_{\nu \geqq L} \nu 2^{-\nu \epsilon / 3} \\
& =5 \omega^{-\epsilon / 6}+12\left[x^{L} \frac{L-(L-1) x}{(1-x)^{2}}\right]_{x=2-\epsilon / 3} \\
& <5 \omega^{-\epsilon / 6}+24 L 2^{-L \epsilon / 3},
\end{aligned}
$$

and from (8) and (6) for $\omega>\omega_{0}(\epsilon)$

$$
\begin{aligned}
& <5 \omega^{-\epsilon / 6}+24\left(\frac{2}{\kappa}\right)^{\epsilon / 3} \frac{\log \kappa}{\log 2}<5 \omega^{-\epsilon / 6}+24 \log \omega\left(\frac{4}{\omega^{1 / 2}}\right)^{\epsilon / 3} \\
& <30 \omega^{-\epsilon / 6} .
\end{aligned}
$$

Since $\epsilon$ is fixed the series (2) converges for $|z|=1$ uniformly. 
Added in proof (September 8, 1948). The fact that also a continuous power series can possess Carleman singularity has been remarked earlier by O. Szász in his paper Über Potenzreihen die in Einheitskreise beschränkte Funktionen darstellen, Math. Zeit. vol. 8 (1920) pp. 222236; his example is also based on Bernstein's polynomials. ${ }^{3}$ As Professor Szász mentioned, the reasoning he and S. Minakshisundaram used in their paper On absolute convergence of multiple Fourier series, Trans. Amer. Math. Soc. vol. 61 (1947) pp. 36-53, and which is different from mine, furnishes also completely elementary examples in the above sense.

UNIVERSITY OF COPENHAGEN 